\begin{tabular}{|c|l|}
\hline Title & Surface acoustic waves in two-dimensional periodic elastic structures \\
\hline Author(s) & Tanaka, Y ukihiro; Tamura, Shin-ichiro \\
\hline Citation & $\begin{array}{l}\text { PHY SICAL REVIEW B, 58(12), 7958.7965 } \\
\text { https://doi.org/L0.1103/PhysRevB.58.7958 }\end{array}$ \\
\hline Issue Date & 1998-09 \\
\hline Doc URL & http://hdl.handle.net/2115/5847 \\
\hline Rights & Copyright $\odot$ 1998 A merican Physical Society \\
\hline Type & article \\
\hline File Information & PRB58 12 .pdf \\
\hline
\end{tabular}

Instructions for use 


\title{
Surface acoustic waves in two-dimensional periodic elastic structures
}

\author{
Yukihiro Tanaka and Shin-ichiro Tamura \\ Department of Applied Physics, Hokkaido University, Sapporo 060, Japan
}

(Received 21 April 1998)

\begin{abstract}
Acoustic waves localized at the surface of two-dimensional (2D) periodic elastic structures, or 2D phononic crystals, are studied theoretically by taking account of the elastic anisotropy of constituent materials. The surface considered is perpendicular to the axis of a periodic array of cylinders embedded in a background material. The dispersion relations of the surface modes are calculated for circular cylinders of AlAs which form a square lattice in a GaAs matrix. The folding and anisotropy of the surface wave branches, as well as the existence of pseudosurface waves, are found. The stop band distributions of the surface, pseudosurface, and bulk waves are plotted in a form relevant for comparison with ultrasound imaging experiments. [S0163-1829(98)00336-1]
\end{abstract}

\section{INTRODUCTION}

There has been a growing interest in recent years in the study of two-dimensional (2D), periodic, dielectric structures called photonic crystals. ${ }^{1,2}$ A major reason for this is the fact that these systems exhibit band gaps (photonic band gaps) which extend throughout the Brillouin zone. The existence of photonic band gaps can lead to a variety of phenomena of both fundamental and practical interest. The absence of electromagnetic modes in a certain frequency range can modify the basic properties of atomic, molecular, and excitonic systems.

The analogy between photons and phonons suggests the consideration of periodic elastic composites of two or more vibrating materials called phononic crystals. So far, several authors have developed for such composites theories of acoustic band structure for bulk vibrations in an isotropic approximation with the wave vector perpendicular to the cylinder axis. ${ }^{3-5}$ Acoustic waves are certainly more complicated than the electromagnetic waves due to the presence of longitudinal vibrations. Also, the presence of elastic anisotropy of both the host and cylinder materials should properly be taken into account.

Of special interest for the phonon case is the prospect of achieving a complete frequency gap, or phonon stop band, for both longitudinal and transverse vibrations. So far, a complete frequency gap has been observed experimentally only for a single mode (longitudinal mode). ${ }^{6}$ However, by choosing constituent materials with large acoustic mismatch, and also by varying the filling fraction of the composites, a phononic band gap extending throughout the Brillouin zone is predicted. ${ }^{5}$ The suppression of the electron-acousticphonon interaction due to the presence of phonon stop bands in 2D superlattices should provide interesting applications.

Another worthwhile subject concerning phononic crystals would be the acoustic mode localized near the truncated surface of the structure. Such localized surface states of photons in 2D periodic dielectric lattices have been studied by Etchegoin and Phillips. ${ }^{7}$ The surfaces they introduced are those parallel to the axes of dielectric rods, and the surface states are found inside the photonic gaps. In the present study we consider a different case, that is, a surface perpendicular to the axes of elastic cylinders forming a 2D lattice, which should be more easily accessible experimentally. ${ }^{8}$ In this case the system occupying $z>0$ is periodic within the flat surface $(z=0)$, which is taken to be the $x-y$ plane and homogeneous in the $z$ direction.

The purpose of the present study is to elucidate theoretically the characteristics of surface acoustic waves in the 2D phononic crystals of the above geometrical configuration. (Hereafter, we call surface acoustic waves simply surface waves.) Full account is taken of the elastic anisotropy of the media composing both cylinders and background. We find that the surface-phonon branch exists below the lowest bulk branch, in general, though it can appear inside the bulk band for a specific direction of propagation. Moreover, the folding effect characteristic of a periodic system can also be found for the surface branches at the Brillouin-zone boundary. Similar results for surface waves have been obtained for 1D phononic crystals, i.e., semi-infinite 1D periodic superlattices with a free surface in the isotropic approximation. ${ }^{9-11}$ An interesting observation in the presence of anisotropy is the fact that the folded surface wave branch continues to exist inside the bulk band as a pseudosurface wave branch after intersection with the dispersion curve of a bulk phonon. We also plot the stop band distribution of both the surface and bulk acoustic waves which would be observable in an ultrasound imaging experiment. ${ }^{8,12,13}$

\section{FORMULATION}

We assume the system to be an elastic continuum composed of a periodic array of cylinders of material $A$ embedded in a background material $B$. Both $A$ and $B$ materials are cubic crystals and elastic anisotropy is fully taken into account. The equation governing the motion of lattice displacement $\mathbf{u}(\mathbf{r}, t)$ of the system is given by

$$
\rho(\mathbf{r}) \ddot{u}_{i}=\partial_{j}\left[c_{i j m n}(\mathbf{r}) \partial_{n} u_{m}\right], \quad(i=1,2,3),
$$

where $\rho(\mathbf{r})$ and $c_{i j m n}(\mathbf{r})$ are the position-dependent mass density and elastic stiffness tensor, respectively, and the 
summation convention over repeated indices is assumed. We try to solve this equation by a standard Fourier expansion method, or we put

$$
\begin{gathered}
\mathbf{u}(\mathbf{r}, t)=\sum_{\mathbf{G}} e^{i(\mathbf{k}+\mathbf{G}) \cdot \mathbf{x}-i \omega t} \mathbf{a}_{\mathbf{G}}(z), \\
\rho(\mathbf{r})=\sum_{\mathbf{G}} e^{i \mathbf{G} \cdot \mathbf{x}} \rho_{\mathbf{G}}, \\
c_{i j m n}(\mathbf{r})=\sum_{\mathbf{G}} e^{i \mathbf{G} \cdot \mathbf{x}} c_{\mathbf{G}}^{i j m n},
\end{gathered}
$$

where $\mathbf{r}=(\mathbf{x}, z)=(x, y, z), \mathbf{k}=\left(k_{x}, k_{y}\right)=\left(k_{1}, k_{2}\right)$ is a Bloch wave vector, and $\mathbf{G}=\left(G_{1}, G_{2}\right)$ is a two-dimensional reciprocal-lattice vector. The Bloch theorem is used for the displacement vector $\mathbf{u}$. Note that $\rho$ and $c_{i j m n}$ do not depend on $z$ because of the homogeneity of the system along the cylinder axis. In order to obtain the surface wave solution we further put

$$
\mathbf{a}_{\mathbf{G}}(z)=\mathbf{A}_{\mathbf{G}} e^{i \lambda z}
$$

Substituting Eqs. (2)-(5) into Eq. (1), we obtain

$$
\left(\begin{array}{ccc}
M_{\mathbf{G}, \mathbf{G}^{\prime}}^{(1)}-\lambda^{2} N_{\mathbf{G}, \mathbf{G}^{\prime}}^{(1)} & L_{\mathbf{G}, \mathbf{G}^{\prime}}^{(1)} & \lambda K_{\mathbf{G}, \mathbf{G}^{\prime}}^{(1)} \\
L_{\mathbf{G}, \mathbf{G}^{\prime}}^{(2)} & M_{\mathbf{G}, \mathbf{G}^{\prime}}^{(2)}-\lambda^{2} N_{\mathbf{G}, \mathbf{G}^{\prime}}^{(2)} & \lambda K_{\mathbf{G}, \mathbf{G}^{\prime}}^{(2)} \\
\lambda J_{\mathbf{G}, \mathbf{G}^{\prime}}^{(1)} & \lambda J_{\mathbf{G}, \mathbf{G}^{\prime}}^{(2)} & M_{\mathbf{G}, \mathbf{G}^{\prime}}^{(3)}-\lambda^{2} N_{\mathbf{G}, \mathbf{G}^{\prime}}^{(3)}
\end{array}\right)\left(\begin{array}{c}
A_{\mathbf{G}^{\prime}}^{1} \\
A_{\mathbf{G}^{\prime}}^{2} \\
A_{\mathbf{G}^{\prime}}^{3}
\end{array}\right)=0
$$

where

$$
\begin{aligned}
& M_{\mathbf{G}, \mathbf{G}^{\prime}}^{(1)}=\omega^{2} \rho_{\mathbf{G}-\mathbf{G}^{\prime}}-\left(k_{1}+G_{1}\right)\left(k_{1}+G_{1}^{\prime}\right) C_{\mathbf{G}-\mathbf{G}^{\prime}}^{11}-\left(k_{2}+G_{2}\right) \\
& \times\left(k_{2}+G_{2}^{\prime}\right) C_{\mathbf{G}-\mathbf{G}^{\prime}}^{44}, \\
& M_{\mathbf{G}, \mathbf{G}^{\prime}}^{(2)}=\omega^{2} \rho_{\mathbf{G}-\mathbf{G}^{\prime}}-\left(k_{2}+G_{2}\right)\left(k_{2}+G_{2}^{\prime}\right) C_{\mathbf{G}-\mathbf{G}^{\prime}}^{11}-\left(k_{1}+G_{1}\right) \\
& \times\left(k_{1}+G_{1}^{\prime}\right) C_{\mathbf{G}-\mathbf{G}^{\prime}}^{44}, \\
& M_{\mathbf{G}, \mathbf{G}^{\prime}}^{(3)}=\omega^{2} \rho_{\mathbf{G}-\mathbf{G}^{\prime}}-(\mathbf{k}+\mathbf{G}) \cdot\left(\mathbf{k}+\mathbf{G}^{\prime}\right) C_{\mathbf{G}-\mathbf{G}^{\prime}}^{44}, \\
& N_{\mathbf{G}, \mathbf{G}^{\prime}}^{(i)}=C_{\mathbf{G}-\mathbf{G}^{\prime}}^{44} \quad(i=1,2), \\
& N_{\mathbf{G}, \mathbf{G}^{\prime}}^{(3)}=C_{\mathbf{G}-\mathbf{G}^{\prime}}^{11}, \\
& L_{\mathbf{G}, \mathbf{G}^{\prime}}^{(1)}=-\left(k_{1}+G_{1}\right)\left(k_{2}+G_{2}^{\prime}\right) C_{\mathbf{G}-\mathbf{G}^{\prime}}^{12} \\
& -\left(k_{1}+G_{1}^{\prime}\right)\left(k_{2}+G_{2}\right) C_{\mathbf{G}-\mathbf{G}^{\prime}}^{44}, \\
& L_{\mathbf{G}, \mathbf{G}^{\prime}}^{(2)}=-\left(k_{2}+G_{2}\right)\left(k_{1}+G_{1}^{\prime}\right) C_{\mathbf{G}-\mathbf{G}^{\prime}}^{12} \\
& -\left(k_{2}+G_{2}^{\prime}\right)\left(k_{1}+G_{1}\right) C_{\mathbf{G}-\mathbf{G}^{\prime}}^{44}, \\
& K_{\mathbf{G}, \mathbf{G}^{\prime}}^{(i)}=-\left(k_{i}+G_{i}\right) C_{\mathbf{G}-\mathbf{G}^{\prime}}^{12}-\left(k_{i}+G_{i}^{\prime}\right) C_{\mathbf{G}-\mathbf{G}^{\prime}}^{44} \quad(i=1,2), \\
& J_{\mathbf{G}, \mathbf{G}^{\prime}}^{(i)}=-\left(k_{i}+G_{i}^{\prime}\right) C_{\mathbf{G}-\mathbf{G}^{\prime}}^{12}-\left(k_{i}+G_{i}\right) C_{\mathbf{G}-\mathbf{G}^{\prime}}^{44} \quad(i=1,2) .
\end{aligned}
$$

In the above equations the Fourier coefficients $C_{\mathbf{G}}^{i j}$ are related to $c_{\mathbf{G}}^{i j m n}$ in a usual manner. Putting, further,

$$
\begin{gathered}
A_{\mathbf{G}}^{i}=\lambda \widetilde{A}_{\mathbf{G}}^{i} \quad(i=1,2), \\
A_{\mathbf{G}}^{3}=\widetilde{A}_{\mathbf{G}}^{3},
\end{gathered}
$$

Eq. (6) is reduced to the generalized eigenvalue equation with respect to $\lambda^{2}$, which determines the spatial variation of the wave with the distance from the surface,

$$
\left(\lambda^{2} P_{\mathbf{G}, \mathbf{G}^{\prime}}-Q_{\mathbf{G}, \mathbf{G}^{\prime}}\right) \tilde{\mathbf{A}}_{\mathbf{G}^{\prime}}=0,
$$

where

$$
\begin{gathered}
P_{\mathbf{G}, \mathbf{G}^{\prime}}=\left(\begin{array}{ccc}
N_{\mathbf{G}, \mathbf{G}^{\prime}}^{(1)} & 0 & 0 \\
0 & N_{\mathbf{G}, \mathbf{G}^{\prime}}^{(2)} & 0 \\
-J_{\mathbf{G}, \mathbf{G}^{\prime}}^{(1)} & -J_{\mathbf{G}, \mathbf{G}^{\prime}}^{(2)} & N_{\mathbf{G}, \mathbf{G}^{\prime}}^{(3)}
\end{array}\right), \\
Q_{\mathbf{G}^{\prime}, \mathbf{G}^{\prime}}=\left(\begin{array}{ccc}
M_{\mathbf{G}, \mathbf{G}^{\prime}}^{(1)} & L_{\mathbf{G}, \mathbf{G}^{\prime}}^{(1)} & K_{\mathbf{G}, \mathbf{G}^{\prime}}^{(1)} \\
L_{\mathbf{G}, \mathbf{G}^{\prime}}^{(2)} & M_{\mathbf{G}, \mathbf{G}^{\prime}}^{(2)} & K_{\mathbf{G}, \mathbf{G}^{\prime}}^{(2)} \\
0 & 0 & M_{\mathbf{G}, \mathbf{G}^{\prime}}^{(3)}
\end{array}\right) .
\end{gathered}
$$

If we truncate the expansions of Eqs. (2)-(4) by choosing $n$ reciprocal-lattice vectors, Eq. (18) gives $3 n$ eigenvalues $\lambda_{l}^{2},(l=1-3 n)$. For the surface wave we are seeking solutions for which the lattice displacement may decay exponentially into the medium $(z>0)$ away from the surface $z=0$. Hence, all $\lambda=\lambda_{l}$ must have a positive imaginary part. If such a set of $\lambda_{l}$ 's are found for a given frequency $\omega$, the displacement vector of the surface wave takes the form

$$
\begin{aligned}
\mathbf{u}(\mathbf{r}, t) & =\sum_{\mathbf{G}}{ }^{\prime} e^{i(\mathbf{k}+\mathbf{G}) \cdot \mathbf{x}-i \omega t} \sum_{l=1}^{3 n} \mathbf{A}_{\mathbf{G}}^{(l)} e^{i \lambda_{l} z}, \\
& =\sum_{\mathbf{G}}{ }^{\prime} e^{i(\mathbf{k}+\mathbf{G}) \cdot \mathbf{x}-i \omega t} \sum_{l=1}^{3 n} X_{l} \boldsymbol{\varepsilon}_{\mathbf{G}}^{(l)} e^{i \lambda l^{z}},
\end{aligned}
$$

where $\boldsymbol{\varepsilon}$ is a unit polarization vector, and the prime of the summation means that the sum over $\mathbf{G}$ is truncated up to $n$.

The surface wave should satisfy the stress-free boundary condition at the surface $z=0$, or 


$$
\left.\left.T_{i 3}\right|_{z=0} \equiv c_{i 3 m n} \partial_{n} u_{m}\right|_{z=0}=0 \quad(i=1,2,3) .
$$

This leads to $3 n$ homogeneous linear equations for $X_{l}$ ( $l$ $=1-3 n)$,

$$
\left(\begin{array}{cccc}
H_{1, \mathbf{G}}^{(1)} & H_{1, \mathbf{G}}^{(2)} & \cdots & H_{1, \mathbf{G}}^{(3 n)} \\
H_{2, \mathbf{G}}^{(1)} & H_{2, \mathbf{G}}^{(2)} & \cdots & H_{2, \mathbf{G}}^{(3 n)} \\
H_{3, \mathbf{G}}^{(1)} & H_{3, \mathbf{G}}^{(2)} & \cdots & H_{3, \mathbf{G}}^{(3 n)}
\end{array}\right)\left(\begin{array}{c}
X_{1} \\
X_{2} \\
\vdots \\
X_{3 n}
\end{array}\right) \equiv \widetilde{H} \mathbf{X}=0
$$

where $\widetilde{H}$ is a $3 n \times 3 n$ matrix, and its elements are

$$
\begin{aligned}
H_{i, \mathbf{G}}^{(l)}=\sum_{\mathbf{G}}{ }^{\prime} C_{\mathbf{G}-\mathbf{G}^{\prime}}^{44}\left[\left(k_{i}+G_{i}^{\prime}\right) \varepsilon_{\mathbf{G}^{\prime}}^{3(l)}+\lambda_{l} \varepsilon_{\mathbf{G}^{\prime}}^{i(l)}\right], \quad(i=1,2), \\
H_{3, \mathbf{G}}^{(l)}=\sum_{\mathbf{G}}^{\prime}\left\{C_{\mathbf{G}^{-} \mathbf{G}^{\prime}}^{11} \lambda_{l} \varepsilon_{\mathbf{G}^{\prime}}^{3(l)}+C_{\mathbf{G}^{-} \mathbf{G}^{\prime}}^{12}\left[\left(k_{1}+G_{1}^{\prime}\right) \varepsilon_{\mathbf{G}^{\prime}}^{1(l)}\right.\right. \\
\left.\left.+\left(k_{2}+G_{2}^{\prime}\right) \varepsilon_{\mathbf{G}^{\prime}}^{2(l)}\right]\right\} .
\end{aligned}
$$

Equation (24) determines the relative weights $X_{l}$ of $3 n$ wave amplitudes if the frequency $\omega$ is correctly chosen. Actually we do not know a priori the eigenfrequency $\omega=\omega_{s}$ of the surface localized acoustic mode, so Eq. (18) and

$$
\operatorname{det}(\widetilde{H})=0
$$

should be solved simultaneously to obtain $\omega_{s}$ and $\lambda_{l}$.

\section{NUMERICAL EXAMPLES}

To develop numerical examples, we specify the phononic crystal more explicitly. We consider the structure where elastic circular cylinders (denoted by $A$ ) of radius $r_{0}$ are embedded periodically in a background material (denoted by $B$ ), forming a square lattice with lattice spacing $a$. In this structure the reciprocal-lattice vector is $\mathbf{G}=\left(2 \pi N_{1} / a, 2 \pi N_{2} / a\right)$ with $N_{1}$ and $N_{2}$ integers, and the Fourier coefficients are given by

$$
\alpha_{\mathbf{G}}=\left\{\begin{array}{lll}
f \alpha_{A}+(1-f) \alpha_{B} & \text { for } & \mathbf{G}=0 \\
\left(\alpha_{A}-\alpha_{B}\right) F(\mathbf{G}) & \text { for } & \mathbf{G} \neq 0,
\end{array}\right.
$$

where $\alpha=\left(\rho, C^{i j}\right), f=\pi r_{0}^{2} / a^{2}$ is the filling fraction which defines the cross-sectional area of a cylinder relative to a unit cell area, and

$$
F(\mathbf{G})=\frac{2 f J_{1}\left(|\mathbf{G}| r_{0}\right)}{|\mathbf{G}| r_{0}},
$$

with $J_{1}(x)$ a Bessel function. In addition, AlAs and GaAs are taken for the cylinder $(A)$ and background $(B)$ materials, respectively. ${ }^{14} \mathrm{We}$ choose the $x-y$ plane (perpendicular to the cylindrical axes) to be parallel to the (001) plane of both AlAs and GaAs, so the fourfold symmetry of the system within the surface is maintained.

\section{A. Dispersion curves}

Figure 1 shows the low-frequency part of the dispersion curves of both the bulk (with wave vectors parallel to the $x-y$ plane) and surface waves along the boundary of the irreduc-

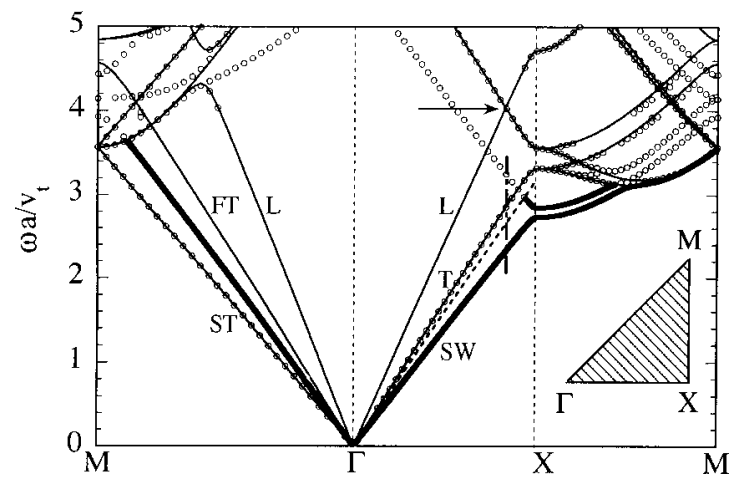

FIG. 1. Dispersion relations of bulk longitudinal $(L)$ and transverse $(T)$ acoustic waves (thin solid lines) and surface acoustic waves (SW, bold solid lines) in a two-dimensional square lattice consisting of AlAs circular cylinders in a GaAs substrate. $\left(v_{T}\right.$ is the transverse sound velocity of bulk GaAs in the [100] direction, i.e., $3.35 \times 10^{5} \mathrm{~cm} / \mathrm{s}$, and $a$ is the lattice spacing.) Also plotted by open circles are the resonances or bulk transverse waves for which the normalized boundary-condition determinants take values smaller than $10^{-4}$. All these branches correspond to waves with a wave vector perpendicular to the cylinder axis. In the $\Gamma-X$ direction fast transverse (FT) and slow transverse (ST) bulk waves are degenerated. The filling fraction $f$ for this figure is 0.564 . The irreducible part of the Brillouin zone is displayed in the inset.

ible part of the Brillouin zone shown in the inset. The value of the filling fraction assumed is $f=0.564$, and the number $n$ of the plane waves kept in the Fourier expansions (2)-(4) is $25\left(N_{1}, N_{2}=0, \pm 1, \pm 2\right)$, for which the convergence of the dispersion curves shown in this figure is satisfactorily attained. We see the existence of the surface wave branch along the $\Gamma-X$ direction ( $k_{x}$ direction), well below the lowest bulk transverse branch. This branch exhibits the folding effect with a frequency gap at the zone-edge $X$ point. The frequency gap continues to exist along the zone boundary almost up to the midpoint of the $X-M$ line.

Along the $\Gamma-M$ direction the surface wave branch appears above the lowest bulk branch, i.e., the slow transverse (ST) branch. In this direction the ST branch is a pure shear mode with horizontal polarization. The surface waves polarized in the sagittal plane are hence completely decoupled from the ST mode, and the relative magnitudes of the surface and bulk wave frequencies are reversed at this isolated direction. No folding effect is seen for the surface mode in this direction because the surface wave branch disappears before reaching the zone boundary by the intersection with the dispersion curves of the bulk modes. At an angle rotated from the $\Gamma-M$ direction the sagittal mode of vibrations couples weakly to the bulk shear mode, leading to the occurrence of resonances (pseudosurface waves) as discussed below.

Complementary information on the surface wave frequencies is obtained from Fig. 2, which displays the angular dependence of dispersion curves at $k a / \pi=0.5$ and $k a / \pi=1$, where $k=|\mathbf{k}|$. Figure 2(a) for $k a / \pi=0.5$ exhibits the dispersion curves of the surface waves very similar to the one obtained on the free surface of a cubic single crystal with anisotropy ratio $\eta=2 C_{44} /\left(C_{11}-C_{12}\right)>1 .{ }^{15}$ Note that the surface wave branch is degenerate to the bulk transverse branch at $45^{\circ}$ (the $\Gamma-M$ direction), and the true surface wave 

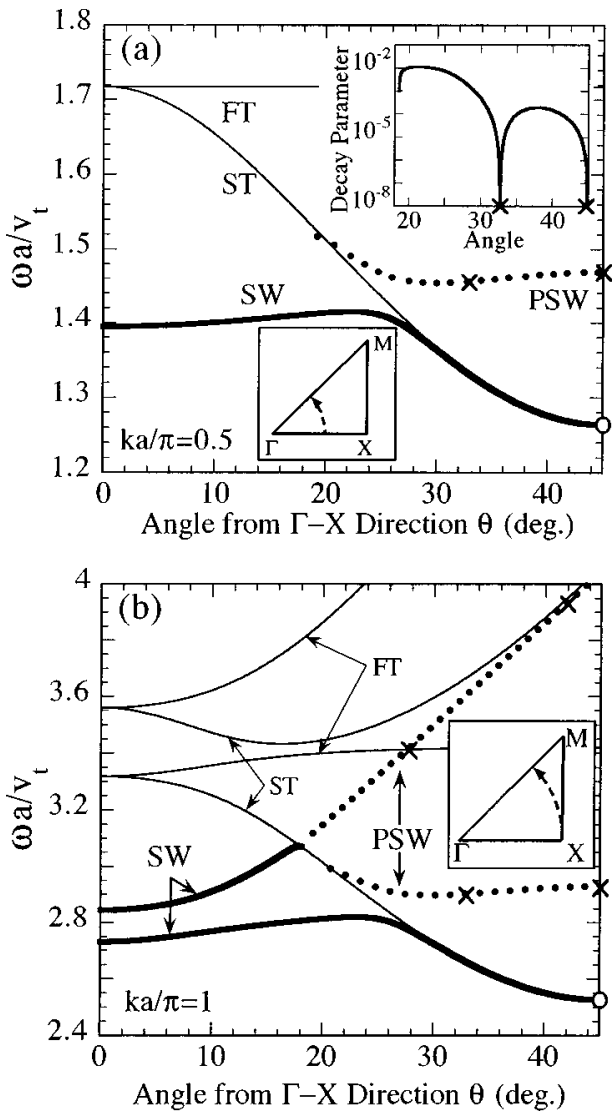

FIG. 2. Angular dependences of the frequencies of the bulk fast transverse (FT) wave, slow transverse (ST) wave (thin solid lines), surface wave (SW, bold solid line), and pseudosurface wave (PSW, dots) (a) at $k a / \pi=0.5$ and (b) at $k a / \pi=1$, i.e., along the dashed line in the Brillouin zone shown in the insets. Crosses on the PSW branches indicate the positions of the true surface waves. The upper inset of (a) shows the decay parameter $\epsilon$ of the PSW's. The filling fraction is $f=0.564$.

at this angle appears at the frequency marked by a cross. At $k a / \pi=1$ [Fig. 2(b)] the situation is more complicated. A folded surface wave branch appears and its frequency increases as the propagation angle $\theta$ increases, but it disappears at the point where the folded branch intersects a bulk transverse branch.

In Fig. 3 the surface wave frequencies $\omega_{s 1}$ and $\omega_{s 2}$, and the magnitude of the frequency gap $\Delta \omega=\omega_{s 2}-\omega_{s 1}$ at the $X$ point are plotted as the function of the filling fraction $f$. Both the surface wave frequencies $\omega_{s 2}$ and $\omega_{s 1}$ at the upper and lower edges of the band gap increase monotonically with $f$, but their difference takes a maximum value at $f=0.564$ (the maximum value of the filling fraction is $\pi / 4=0.7854$ for the square lattice). This value of the filling fraction is chosen in plotting the dispersion curves of Fig. 1. Similar filling fraction dependences of the width of frequency gap are also seen for bulk waves.

In Fig. 4 we illustrate the profiles of the displacement amplitudes of the surface mode at the upper edge $\omega_{s 2}$ and at the lower edge $\omega_{s 1}$ of the band gap at the $X$ point. (No shear component with horizontal polarization exists in the surface wave at this point.) The selected position is the center of the cylinder consisting of AlAs. At $\omega=\omega_{s 2}$ the wave is polarized almost perpendicular to the surface (transverselike) and

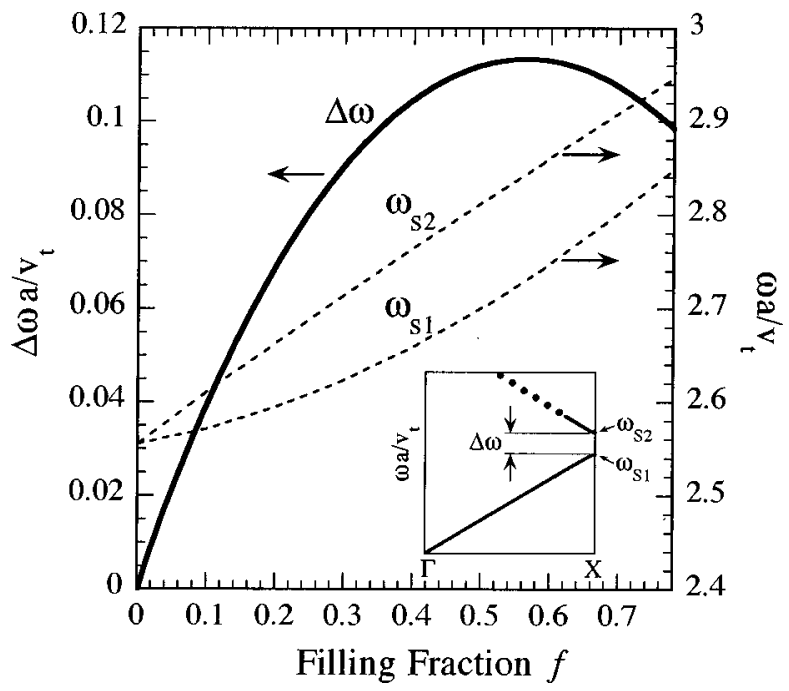

FIG. 3. Magnitude of the frequency gap $\left(\Delta \omega=\omega_{s 2}-\omega_{s 1}\right)$ of the surface mode at the $X$ point vs filling fraction $f$. The frequencies $\omega_{s 2}$ at the upper edge and $\omega_{s 1}$ at the lower edge of the frequency gap at the $X$ point are also plotted. The inset shows the schematic of the dispersion curves of the surface mode along the $\Gamma-X$ direction (solid lines). The maximum value of the filling fraction is $\pi / 4$ $=0.7854$ for the square lattice.
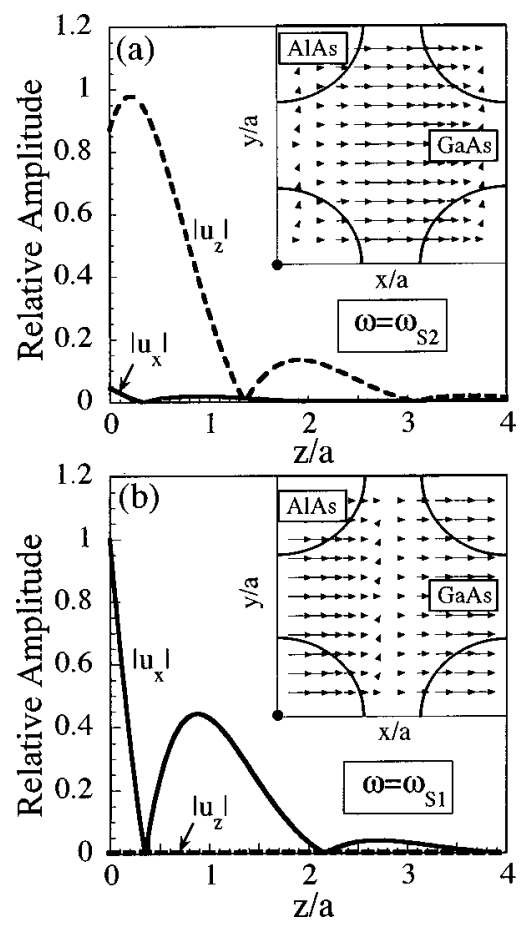

FIG. 4. The lattice displacements $u_{x}$ (parallel to the wave vector along the $x$ axis) and $u_{z}$ (normal to the surface) at the center of the cylinder vs distance from the surface, (a) at the upper edge ( $\omega$ $\left.=\omega_{s 2}\right)$ and (b) at the lower edge $\left(\omega=\omega_{s 1}\right)$ of the frequency gap at the $X$ point. (The component of the lattice displacement along the $y$ axis is zero.) The inset shows the polarizations of the surface acoustic waves in a unit cell at the $X$ point. The length of the horizontal arrow is proportional to $\left|u_{x}\right| /\left(u_{x}^{2}+u_{z}^{2}\right)^{1 / 2}$, which measures the magnitude of the lattice displacement along the surface. The propagation direction is along the $x$ axis. 
at $\omega=\omega_{s 1}$ the wave is longitudinal. However, additional global pictures illustrated in the insets show that at the upper edge $\omega_{s 2}$ the displacement vector is predominantly longitudinal in the heavier GaAs substrate, and that it is polarized rather transversely at the lower edge $\omega_{s 1}$.

Here we note that the folded surface wave branch in the $\Gamma-X$ direction disappears at the point where the upgoing surface wave branch intersects the dashed line, i.e., before the surface wave branch intersects the bulk transverse wave propagating parallel to the surface. The reason is understood in terms of Fig. 5 which shows the lower part of the dispersion curves in the $\Gamma-X$ direction as well as the $k_{x}-k_{z}$ section of the constant-frequency surfaces ( $\omega$ surfaces) of the lowest three bulk branches. At a given low value of the frequency the wave number of the surface wave indicated by $k_{S W}$ exists along the $k_{x}$ axis outside three $\omega$ surfaces of the bulk modes [Figs. 5(a) and 5(b)]. The key observation is the fact that the outermost curve of the slow transverse wave is deformed considerably from a circle due to the anisotropy of the underlying lattice. Thus the tangential line of this curve parallel to the $k_{z}$ axis intersects the $k_{x}$ axis at the point $k_{S T}$ larger than $k_{T}$. The relation between $\omega$ and $k_{S T}$ defines the dashed line in Figs. 1 and 5(a). When the wave number $k_{S W}$ becomes smaller than $k_{S T}$ we can find a real wave vector $\mathbf{K}$ $=\left(k_{S W}=k_{P S W}, k_{z}\right)$ pointing inside the substrate [Fig. 5(c)]. This means if the surface wave couples to the ST mode it is attenuated by radiating the acoustic energy into the bulk of the system. The existence of such a coupling leads to the appearance of new pseudosurface waves or resonances in the present system considered.

We are also interested in how far the surface wave frequency is separated from the frequency of the lowest bulk wave with the same wave vector. This separation is sensitive to the acoustic mismatch between the materials composing the cylinders and background, and also to the filling fraction $f$. To obtain an idea of this, we have changed the density $\rho_{A}$ of the cylinders relative to $\rho_{B}\left(=\rho_{G a A s}\right)$ of the background material, but kept their elastic constants the same. The results at the $X$ point are plotted in Fig. 6. A remarkable feature is the fact that the upper-edge frequency $\omega_{s 2}$ of the folded branch of the surface mode depends strongly on $\rho_{A} / \rho_{B}$. No folded branch appears for $\rho_{A} / \rho_{B} \leqslant 0.3$.

\section{B. Pseudosurface waves}

Figure 7(a) displays the magnitude of boundary-condition determinant $\mid$ det $\widetilde{H} \mid$ along the vertical dot-dashed line of Fig. 1. In addition to the deep minimum associated with the surface wave, two local minima of $|\operatorname{det} \widetilde{H}|$ are found. The minimum at the lower frequency is the bulk transverse wave, and the one at the higher frequency corresponds to the resonance which Every studied for the surfaces of bulk solids. ${ }^{16}$

The open circles of Fig. 1 indicate the positions of the local minima of $|\operatorname{det} \widetilde{H}|$ satisfying $|\operatorname{det} \widetilde{H}| /|\operatorname{det} \widetilde{H}|_{\max }$ $<10^{-4}$ for each $k$, where $|\operatorname{det} \widetilde{H}|_{\max }$ is the maximum value of $\mid$ det $\widetilde{H} \mid$ in the range $0<\omega a / v_{t}<5 .{ }^{17}$ These minima occur either on the bulk transverse branches or along the lines above the lowest bulk wave branches, i.e., inside the bulk band. In the $\Gamma-X$ direction, for example, we find the resonances along the line continuing from the folded branch of
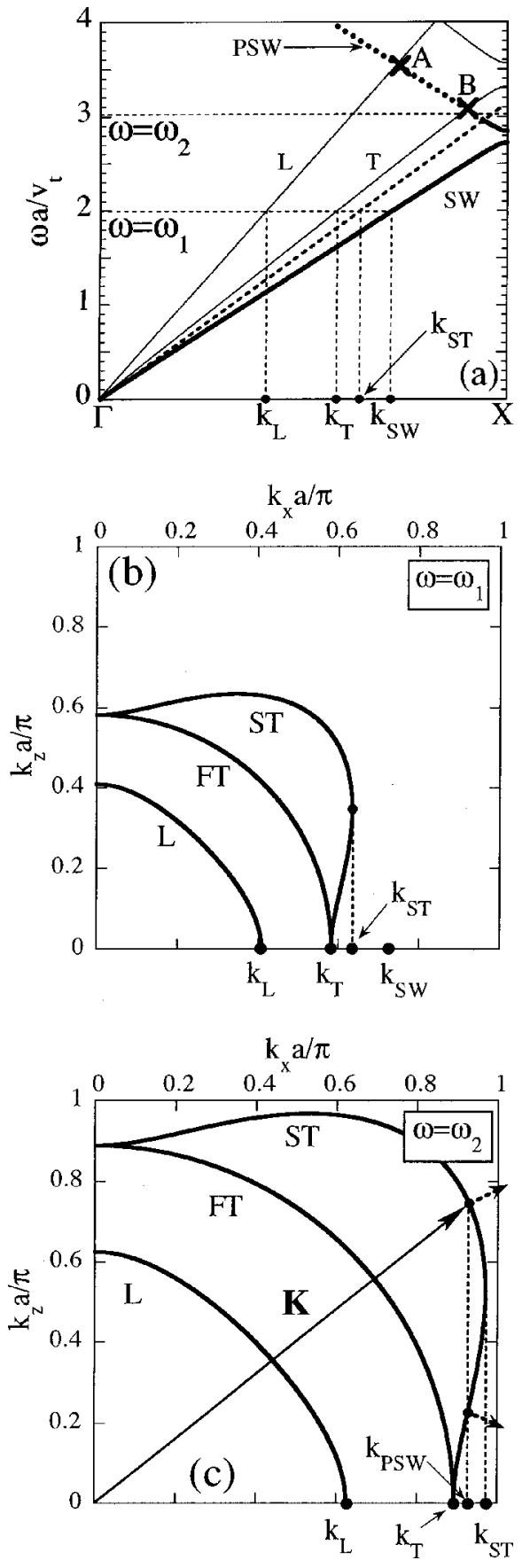

FIG. 5. (a) Magnification of the lower part of the dispersion relations in the $\Gamma-X$ direction of Fig. 1 . The points $k_{L}, k_{T}, k_{S T}$, and $k_{S W}$ indicate the wave numbers in the $x-y$ plane of the bulk and surface waves of a given frequency $\omega$. (b) The section of the constant-frequency $\left(\omega=\omega_{1}\right)$ surfaces of the bulk waves by the $k_{x}-k_{z}$ plane. The wave numbers $k_{L}, k_{T}, k_{S T}$, and $k_{S W}$ correspond to those shown in (a). (c) The section of the constant-frequency $\left(\omega=\omega_{2}\right)$ surfaces of the bulk waves by the $k_{x}-k_{z}$ plane. The wave numbers $k_{L}, k_{T}, k_{P S W}$, and $k_{S T}$ are defined by the intersections of the dispersion curves with the horizontal dashed line $\left(\omega=\omega_{2}\right)$ in (a). We can find a real wave vector $\mathbf{K}$ whose component parallel to the surface is $k_{P S W}$ and the corresponding group-velocity direction (outward normal of the surface plotted by the short dashed line) points in the direction $z>0$. This means that the PSW branch shown by the dotted line of (a) radiates the energy into the bulk of the system. 


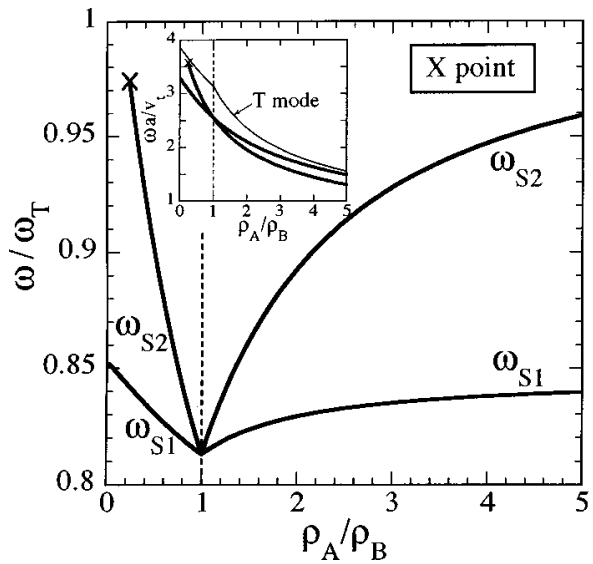

FIG. 6. Surface wave frequencies $\omega_{s 1}$ and $\omega_{s 2}$ at the $X$ point vs the relative density of constituent materials. Frequencies are normalized by $\omega_{T}$, i.e., the frequency of the lowest transverse $(T)$ branch at the same point. The cross shows the point at which the upper frequency $\omega_{s 2}$ touches the bulk transverse branch, and no folded branch appears for $\rho_{A} / \rho_{B} \leqslant 0.3$. The inset shows a similar plot, but the frequency is not normalized by $\omega_{T} \cdot \rho_{B}=\rho_{\text {GaAs }}$, and the elastic constants of GaAs are assumed for both the cylinder and substrate.

the surface mode and their frequencies are inside the bulk band. Hence they possess bulk wave components, and the amplitudes at the surface should decay as they propagate along the surface, as noted in Sec. III A. This can be explicitly seen from the fact that the dip of the boundary-condition determinant goes down further (essentially to zero) if we add a small imaginary part to the two-dimensional wave vector $\mathbf{k}$ as $\mathbf{k}(1+i \epsilon)$, where $\epsilon$ is a real, positive number and its magnitude is typically $\sim 10^{-2}$ or less [see Fig. 7(b)]. In a semiinfinite solid with a free surface, these waves are known as pseudosurface waves (PSW's). ${ }^{15,18,19}$

To obtain the PSW's solutions we have to assign, at the same time, a negative imaginary part (instead of a positive imaginary part for the surface modes) to one or two $\lambda_{l}$ 's of the dominant partial waves. This is required because the decay of the amplitude along the surface should be balanced by the growth of the amplitude into the bulk of the system. In the present work we call the resonances PSW's if the boundary-condition determinant decreases to a value as large as that of the surface waves by introducing the decay parameter $\epsilon$ as well as the bulk wave components radiating the acoustic energy into the substrate. Because of the small decay parameter $\epsilon$, PSW's can propagate along the surface almost unattenuated, and should be observed experimentally just like true surface waves. ${ }^{18}$

In Fig. 8 we show the decay parameter $\epsilon$ of PSW's in the $\Gamma-X$ direction. The magnitude is about $1 \times 10^{-2}$ or less, as expected, so they should behave like surface waves for a traveling distance of ten wavelengths or longer. An interesting observation is that $\epsilon$ vanishes at $k a / \pi \sim 0.73$ (point $A$ ) and $\sim 0.9$ (point $B$ ). At the latter value of $k$ the PSW is degenerate to the bulk transverse wave (see Fig. 1). However, at the former value of $k$ the PSW becomes a true surface wave with the polarization vector out of the symmetry plane. Such a surface wave in a semi-infinite solid is called a

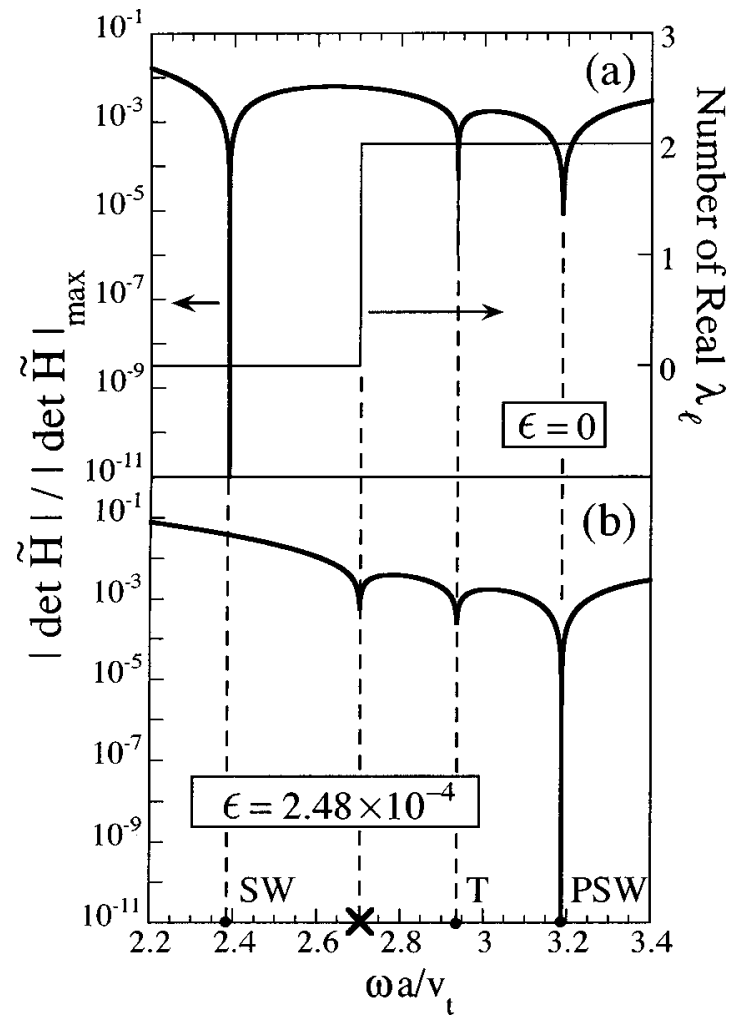

FIG. 7. The normalized magnitude of the boundary condition determinant $|\operatorname{det} \widetilde{H}|$ vs frequency along the dot-dashed line ( $k a / \pi=0.857$ along the $\Gamma-X$ direction) in Fig. 1. (a) For the real wave vector parallel to the surface, with $\epsilon=0$. (b) For the complex wave vector parallel to the surface, i.e., $\epsilon=2.48 \times 10^{-4}$. The frequencies at which the four branches intersect the dot-dashed line (Fig. 1) are labeled. (The cross indicates the frequency at which the dashed line of Fig. 1 intersects the dot-dashed line.) The number of real $\lambda_{l}$ (the wave number normal to the surface) is also shown in (a).

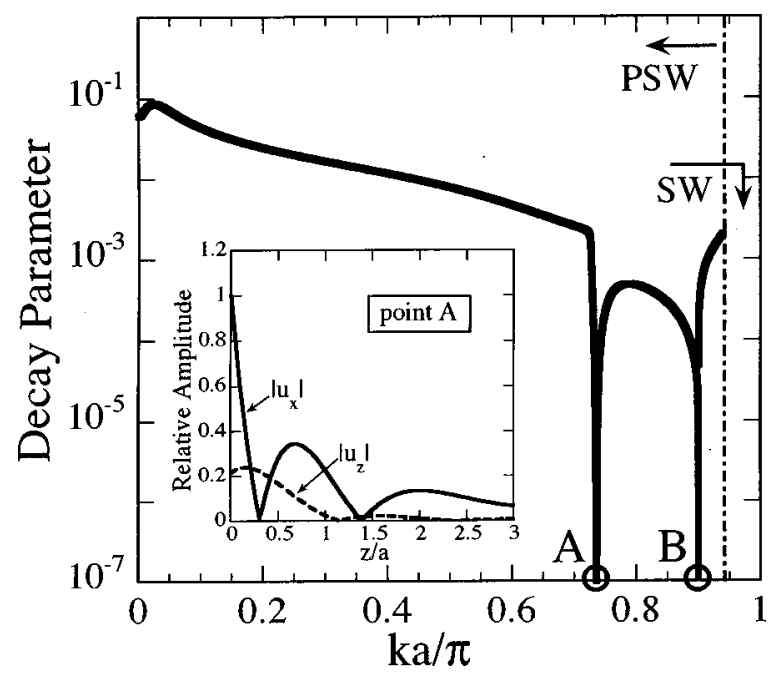

FIG. 8. Decay parameter $\epsilon$ of the pseudosurface waves (PSW's) along the $\Gamma-X$ direction. For the value of $k$ at which $\epsilon$ vanishes (point $A$ ), PSW becomes a true surface wave (secluded supersonic surface wave). At point $B$, PSW is degenerate to the bulk transverse wave propagating along the surface. The lattice displacements vs distance from the surface at point $A$ are plotted in the inset. 
secluded supersonic surface wave. Secluded supersonic surface waves in a germanium surface were recently studied by Maznev and Every. ${ }^{20}$

Another characteristic feature of the PSW's is found by changing the propagation angle $\theta$ for a fixed magnitude of the wave vector $\mathbf{k}$. The angular dependences of the dispersion relations for $k a / \pi=0.5$ and 1 are shown in Fig. 2 by dotted lines. In particular, two PSW branches can be seen in Fig. 2(b), plotted for the larger value of $k$. The lower line [also the line in Fig. 2(a)] is quite similar to the one in the free surface of bulk GaAs, ${ }^{21}$ and the upper branch seems to continue from the folded surface wave branch. The magnitude of the decay parameter $\epsilon$ is again about $1 \times 10^{-2}$ or less, and the secluded supersonic surface waves are found in the PSW branches (crosses in Fig. 2), as explicitly shown in the inset of Fig. 2(a).

\section{Stop band distribution}

A possible experiment to verify the propagation of the surface waves and the PSW's would make use of point- or line-focus ultrasonic immersion transducers, which act as a transmitter and a receiver for an acoustic signal. This ultrasonic method has been developed by Vines, Hauser, and Wolfe for characterizing the propagation of coherent surface acoustic waves in anisotropic media. ${ }^{13}$ By rotating the sample about an axis normal to the surface, the propagation of waves along any direction in the surface can be recorded. When point-focus transducers are used, ultrasonic waves with a broad angular distribution of wave vectors are excited, so the measurement reveals the acoustic energy flux or group velocity along a given direction. On the other hand, ultrasonic waves with a well-defined wave vector are produced if line-focus transducers are employed. In these experiments the occurrence of band gaps in the transmission spectrum can be detected.

In view of these possible experiments, we in Fig. 9 have plotted the distributions of the lowest four frequency gaps of the surface, pseudosurface, and bulk waves as a function of wave-vector direction. The lowest distributions around $\theta=0^{\circ}$ and their equivalent directions are the stop bands of surface waves, and the hatched regions continuing from these distributions are the stop bands of PSW's. The upper distributions are the stop bands of the bulk waves.

An interesting observation is the fact that the width of the stop band labeled $L-T$ vanishes in the $\Gamma-X$ direction. The corresponding frequency is shown by the horizontal arrow in Fig. 1, where the dispersion curves of the longitudinal and (folded) transverse branches intersect each other. As the propagation direction is rotated from the $\Gamma-X$ direction, the frequency gap is open and increases due to the mixing of the polarization vectors of these modes (see the inset of Fig. 9). Thus this stop band exists inside the Brillouin zone, and is physically interpreted as an intermode Bragg reflection of acoustic waves, which has been predicted theoretically and also verified experimentally in one-dimensional periodic superlattices. ${ }^{22-24}$ The other two kinds of stop bands of the bulk transverse and surface waves are due to the intramode Bragg reflections characteristic of a periodic system.

All these stop bands are related to the waves with wave vectors parallel to the surface, and have sizable magnitudes.

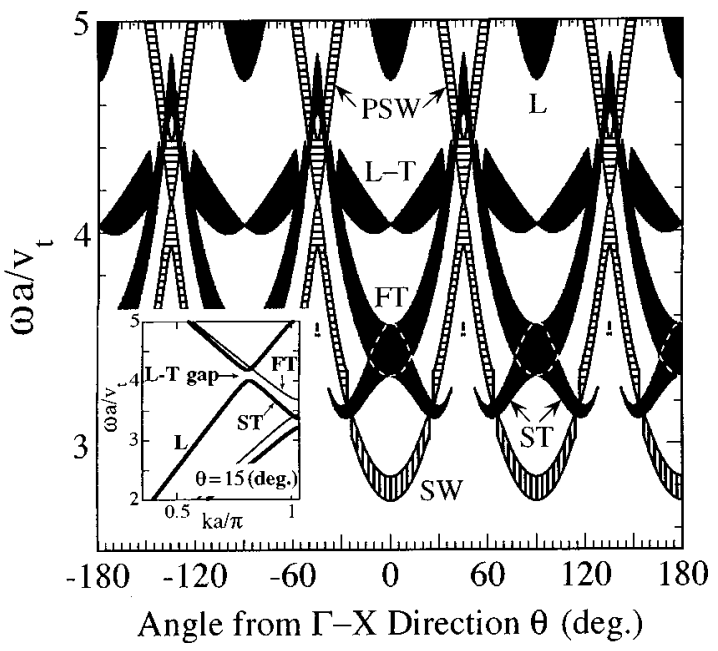

FIG. 9. Distributions of the stop bands of the bulk waves [longitudinal $(L)$, fast transverse (FT), slow transverse (ST) waves, and the coupled longitudinal and transverse $(L-T)$ waves, smeared regions], the surface waves (SW, vertically hatched regions), and pseudosurface waves (PSW, horizontally hatched regions). The FT and ST stop bands are overlapped in the regions surrounded by the dashed lines. The horizontal axis is the angle in wave-vector space. The inset shows the relevant part of the dispersion relations at $\theta=15^{\circ}$, where the intrazone $L-T$ stop band is created.

Hence they should be observed in a wave-vector-scanning technique in which an ultrasonic wave is focused on a line using a cylindrical acoustic lens and a receiving transducer is cylindrically focused on a parallel line some distance away on the surface. Such a preliminary experiment with a polymer filling periodically drilled holes in an aluminum substrate was done by Vines and Wolfe, ${ }^{8}$ and the existence of a stop band distribution similar to that in Fig. 8 has been observed in the transmitted acoustic signal.

\section{CONCLUDING REMARKS}

In the present work we have calculated the dispersion relations of surface and pseudosurface acoustic waves in two-dimensional phononic crystals consisting of periodic arrays of circular cylinders embedded in a background substance. The surface is a plane perpendicular to the cylinder axes, and we find folding effects of the surface-wave dispersion relations which are characteristic of a periodic system. The folded dispersion curves continue to go up into the bulk band as PSW branches. A similar continuation of the PSW branch from the surface wave branch was recently also predicted for $1 \mathrm{D}$ periodic superlattices with a free surface. ${ }^{21}$ The secluded supersonic surface waves are found at some isolated points on the pseudosurface branches. The distributions of frequency gaps, i.e., stop bands, of surface acoustic waves are well separated from those of bulk waves, and they should be observed, for example, by ultrasound imaging experiments.

In the present calculation a square lattice is considered for the configuration of cylinders in the host material. This lattice structure is accommodated by the fourfold symmetry in the surfaces under consideration owing to the materials (cubic crystals) taken for the cylinders and background. However, other lattice structures with lower symmetry such as 
hexagonal, graphite structure, etc., would be worth studying. It would be also interesting to study the surface acoustic waves in a $2 \mathrm{D}$ phononic crystals with larger acoustic mismatch, in which complete frequency gaps common to three bulk modes are formed in the lower part of the dispersion relations.

A preliminary ultrasound imaging experiment with $2 \mathrm{D}$ phononic crystals ${ }^{8}$ suggests at least two subjects for further study. The first one is the effect of liquid loading on the surface of phononic crystals. In the imaging experiment immersion transducers are used to generate and detect ultrasound signals, so the sample surface is covered with liquid. The second one is the frequency dependence of the elastic constants of the material composing the cylinders. In the experiment a polymer is filled in the circular cylinders drilled in a metallic substrate. The elastic constants of polymers are generally frequency dependent in the $\mathrm{MHz}$ range appropriate to the experiment, and at the same time the ultrasonic absorption is very large. We plan to extend our theory to such systems with frequency-dependent, complex elastic constants.

\section{ACKNOWLEDGMENTS}

The authors are grateful to R. E. Vines and J. P. Wolfe for sending preliminary data of their imaging experiment. They also acknowledge $\mathrm{O}$. B. Wright for a critical reading of the manuscript. This work was supported in part by a Grant-inAid for Scientific Research from the Ministry of Education, Science and Culture of Japan (Grant No. 09640385).
${ }^{1}$ Photonic Band Gaps and Localization, edited by C. M. Soukoulis (Plenum, New York, 1993).

${ }^{2}$ J. D. Joannopoulos, R. D. Meade, and J. N. Winn, Photonic Crystals (Princeton University Press, Princeton, 1995).

${ }^{3}$ M. S. Kushwaha, P. Halevi, L. Dobrzynski, and B. DjafariRouhani, Phys. Rev. Lett. 71, 2022 (1993).

${ }^{4}$ M. S. Kushwaha, P. Halevi, G. Martinez, L. Dobrzynski, and B. Djafari-Rouhani, Phys. Rev. B 49, 2313 (1994).

${ }^{5}$ M. Sigalas and E. N. Economou, Solid State Commun. 86, 141 (1993).

${ }^{6}$ F. R. Montero de Espinosa, E. Jimenez, and M. Torres, Phys. Rev. Lett. 80, 1208 (1998).

${ }^{7}$ P. Etchegoin and R. T. Phillips, Phys. Rev. B 53, 12674 (1996).

${ }^{8}$ R. E. Vines and J. P. Wolfe (unpublished).

${ }^{9}$ R. E. Camley, B. Djafari-Rouhani, L. Dobrzynski, and A. A. Maradudin, Phys. Rev. B 27, 7318 (1983).

${ }^{10}$ B. Djafari-Rouhani, L. Dobrzynski, O. Hardouin Duparc, R. E. Camley, and A. A. Maradudin, Phys. Rev. B 28, 1711 (1983).

${ }^{11}$ B. Djafari-Rouhani, A. A. Maradudin, and R. F. Wallis, Phys. Rev. B 29, 6454 (1984).

${ }^{12}$ R. E. Vines, S. Tamura, and J. P. Wolfe, Phys. Rev. Lett. 74, 2729 (1995); 75, 1873(E) (1995).
${ }^{13}$ R. E. Vines, M. R. Hauser, and J. P. Wolfe, Z. Phys. B 98, 255 (1995).

${ }^{14}$ We used the elastic constants $C_{A}^{11}=12.02, C_{A}^{12}=5.70$, and $C_{A}^{44}$ $=5.89$ (in units of $10^{11} \mathrm{dyn} / \mathrm{cm}^{2}$ ) and mass density $\rho_{A}$ $=3.76 \mathrm{~g} / \mathrm{cm}^{3}$ for AlAs, and $C_{B}^{11}=11.88, C_{B}^{12}=5.38$, and $C_{B}^{44}$ $=5.94$ (in units of $10^{11} \mathrm{dyn} / \mathrm{cm}^{2}$ ) and mass density $\rho_{B}$ $=5.36 \mathrm{~g} / \mathrm{cm}^{3}$ for GaAs.

${ }^{15}$ G. W. Farnell, in Physical Acoustics VI, edited by W. P. Mason and R. N. Thurston (Academic, New York, 1970), p. 109.

${ }^{16}$ A. G. Every, Phys. Rev. B 33, 2719 (1986).

${ }^{17}$ For surface wave solutions $|\operatorname{det} \widetilde{H}| /|\operatorname{det} \widetilde{H}|_{\text {max }}$ decreases down to $10^{-13}-10^{-14}$.

${ }^{18}$ F. R. Rollins, Jr., T. C. Lim, and G. W. Farnell, Appl. Phys. Lett. 12, 236 (1968).

${ }^{19}$ T. C. Lim and G. W. Farnell, J. Acoust. Soc. Am. 45, 845 (1969).

${ }^{20}$ A. A. Mazunev and A. G. Every, Phys. Lett. A 197, 423 (1995).

${ }^{21}$ T. Aono and S. Tamura, Phys. Rev. B (to be published 15 August 1998).

${ }^{22}$ S. Tamura and J. P. Wolfe, Phys. Rev. B 35, 2528 (1987).

${ }^{23}$ D. C. Hurley, S. Tamura, J. P. Wolfe, and H. Morkoc, Phys. Rev. Lett. 58, 2446 (1987).

${ }^{24}$ S. Tamura, D. C. Hurley, and J. P. Wolfe, Phys. Rev. B 38, 1427 (1988). 